\title{
Using Guided Filtering to Improve Gram-Schmidt Based Pansharpening Method for GeoEye-1 Satellite Images
}

\author{
$\mathrm{Xu} \mathrm{Li}{ }^{1}$, Yiming Zhang ${ }^{1}$, Yanan Gao ${ }^{1}$, Shigang Yue ${ }^{2}$ \\ 1. School of Electronics and Information, Northwestern Polytechnical University, China \\ 2. School of Computer Science, University of Lincoln, Lincoln, LN6 7TS UK \\ E-mail:lixu@nwpu.edu.cn,syue@lincoln.ac.uk
}

\begin{abstract}
Gram-Schmidt based spectral pansharpening is a well-known scheme for fusion of panchromatic (PAN) and multispectral (MS) images. However, it relies more on the relative spectral responses between the PAN and MS sensors, which may easily suffer from spectral distortions. To deal with this problem, we present a new Gram-Schmidt based pansharpening method using guided filtering to improve the sharpened quality. The spatial information of PAN image including details and structures are effectively extracted through guided filtering and then injected into the MS imagery. The experiment is carried out on GeoEye-1 satellite images. Visual and objective analysis show that our method can produce high-quality pansharpened results and outperform some existing methods.
\end{abstract}

Keywords: pansharpening; Gram-Schmidt transformation; guided filter

\section{Introduction}

With the fast development of spaceborne imaging sensors, many satellites provide many high-resolution remote sensing images. Some in-orbit high-resolution commercial satellites, such as IKONOS, QuickBird, GeoEye-1 and WorldView-2 can capture both the panchromatic (PAN) image and multispectral (MS) images at almost the same time. Due to the physical constraints, the PAN band without spectral diversity covers wide spectral range (usually from visual to near infrared) and has very high spatial resolution. On the contrary, MS imagery has lower spatial resolution but higher spectral resolution. The satellite images that simultaneously offer high spatial and spectral resolutions are often required in many remote sensing applications. Pansharpening has been an important tool to meet this goal, whose aim is to enhance the spatial resolution of MS images with the aid of PAN spatial information. In recent twenty years, a wide variety of pansharpening methods have been developed. Gram-Schmidt (GS) spectral sharpening is one of the most popular methods, which was invented by Laben and Brower in 1998 and patented by Eastman Kodak [1]. Famous commercial software such as ENVI and ERDAS have integrated GS method in their sharpening modules. In the forward GS transformation, GS method builds the low-resolution version of the Pan $\left(\mathrm{P}_{\mathrm{L}}\right)$ image by simply averaging all the MS images, which causes the obvious spectral distortions in the sharpened results. In order to improve the GA method, Bruno Aiazzi et al. considered the relative spectral response between the PAN and MS bands and proposed an adaptive GS method (named GSA) in which the $\mathrm{P}_{\mathrm{L}}$ image is estimated through multivariate regression [2]. Actually, the spectral responses vary with different imaging sensors, and the spectral response of PAN band may not cover all the MS bands. It is not necessary for PAN and MS bands to have definitely mutual spectral response relations. Motivated by this, we propose an improved GS pansharpening method regardless of the spectral responses. To verify its effectiveness, the experiments are carried out on GeoEye-1 data set. The subjective and objective evaluations show that the proposed method can produce high quality results and outperforms GS, GSA, and some other methods.

\section{Guided filter and GS method}

\subsection{Guided filter}

Guided filter is an edge-preserving smoothing operator and it can transfer the structures of the guidance image to the filtering output [3]. It is both effective and efficient in a great variety of computer vision and computer graphics applications, including edge-aware smoothing, detail enhancement, image matting, etc. According to the definition in [3], it is assumed that the filtering output $\boldsymbol{Q}$ is a linear transform of the guidance image $\boldsymbol{G}$ in a window $w_{k}$ centered at the pixel $k$ : 


$$
\boldsymbol{Q}_{i}=a_{k} \boldsymbol{G}_{i}+b_{k}, \forall i \in w_{k}
$$

where $\left(a_{k}, b_{k}\right)$ are some linear coefficients assumed to be constant in $w_{k}$, and $w_{k}$ is a square window with a radius $r$. The linear coefficients $\left(a_{k}, b_{k}\right)$ is determined by modeling the output $\boldsymbol{Q}$ as the input $\boldsymbol{P}$ subtracting some unwanted components $N$ like noise or textures:

$$
\boldsymbol{Q}_{i}=\boldsymbol{P}_{i}-\boldsymbol{N}_{i}
$$

Then the $a_{k}$ and $b_{k}$ can be estimated by seeking a cost function in the window $w_{k}$ to minimizing the difference between $\boldsymbol{Q}$ and $\boldsymbol{P}$ while maintaining the linear model:

$$
E\left(a_{k}, b_{k}\right)=\sum_{i \in w_{k}}\left(\left(a_{k} \boldsymbol{G}_{i}+b_{k}-\boldsymbol{P}_{i}\right)^{2}+\varepsilon a_{k}^{2}\right)
$$

in which $\varepsilon$ is a regularization parameter selected by the users. Eq. (3) is the linear ridge regression and its solution is given by

$$
\begin{gathered}
a_{k}=\frac{\frac{1}{|w|} \sum_{i \in w_{k}}\left(G_{i} P_{i}-\mu_{k} \bar{p}_{k}\right)}{\sigma_{k}^{2}+\varepsilon} \\
b_{k}=\bar{p}_{k}-a_{k} \mu_{k}
\end{gathered}
$$

Here, $\mu_{k}$ and $\sigma_{k}$ are the mean and variance of $\boldsymbol{G}$ in $w_{k}$, respectively. $|w|$ is the number of all the pixels in $w_{k}$ and $\bar{p}_{k}$ is the mean of $\boldsymbol{P}$ in $w_{k}$. Since all the overlapping windows $w_{k}$ covering the pixel $i$ has different values of $\left(a_{k}, b_{k}\right), \boldsymbol{Q}_{i}$ in (1) is different. To solve this problem, averaging all the possible $\left(a_{k}, b_{k}\right)$ values and estimating the filtering output is as follows

$$
\boldsymbol{Q}_{i}=\bar{a}_{i} \boldsymbol{G}_{i}+\bar{b}_{i}
$$

in which $\bar{a}_{i}=\frac{1}{|w|} \sum_{k \in w_{i}} a_{k}, \bar{b}_{i}=\frac{1}{|w|} \sum_{k \in w_{i}} b_{k}$. Equations (4), (5), and (6) are the definition of the guided filter. In this paper, we denote $G F_{r, \varepsilon}(\boldsymbol{G}, \boldsymbol{P})$ as the guided filtering operation in which $r$ and $\varepsilon$ represent the radius of the filtering window and the blurring degree, respectively. $\boldsymbol{G}$ and $\boldsymbol{P}$ represent the guidance image and input image, respectively.

\subsection{GS method}

In the GS method, as described by its inventors [1], the spatial resolution of MS image is enhanced by merging the high-resolution PAN image with the low spatial resolution MS bands. The main steps of this method are the following.

1) A lower spatial resolution PAN $\left(\boldsymbol{P}_{\boldsymbol{L}}\right)$ image is simulated at the same scale of PAN.

2) The forward GS transformation (GST) is performed on the $\boldsymbol{P}_{\boldsymbol{L}}$ image together with all the lower spatial resolution MS bands upscaled to the same scale of PAN. The $\boldsymbol{P}_{\boldsymbol{L}}$ is employed as the first band in the GST.

3) The statistics of the PAN image is adjusted to match the statistics of the first transform band $\left(\boldsymbol{G} \boldsymbol{S}_{1 \mathrm{LR}}\right)$ that results from the GST to produce a modified PAN image.

4) The modified PAN image takes place the $\boldsymbol{G} \boldsymbol{S}_{1 \mathrm{LR}}$ to produce a new set of transform bands.

5) The inverse GST is performed on the new set of transform bands to produce the enhanced spatial resolution MS images.

Typically, the $\boldsymbol{P}_{\boldsymbol{L}}$ is obtained as the pixel average of the MS bands. This average way is simple, but spectral distortions with respect to the resampled MS image may occur because the average of the MS bands is not likely to have the same radiometry as the PAN image. Alternatively, it is user's task to build the $\boldsymbol{P}_{\boldsymbol{L}}$ that is usually obtained by preliminarily low-pass filtering which may suffer from a lower sharpness and spatial enhancement due to improper digital filtering of the PAN image.

\section{The proposed method}

To reduce the spectral distortions and improve the sharpened quality, we proposed a novel method which takes the advantages of guided filtering and GS transformation. Fig. 1 shows the schematic diagram. All the steps of the proposed method are described as follows:

1) The original MS bands are resampled to the same scale as the PAN through bicubic interpolation. The upscaled low-resolution MS images are denoted as $\boldsymbol{L} \boldsymbol{R} \boldsymbol{M}_{i}$, where $i=1 \ldots \mathrm{N}$. 


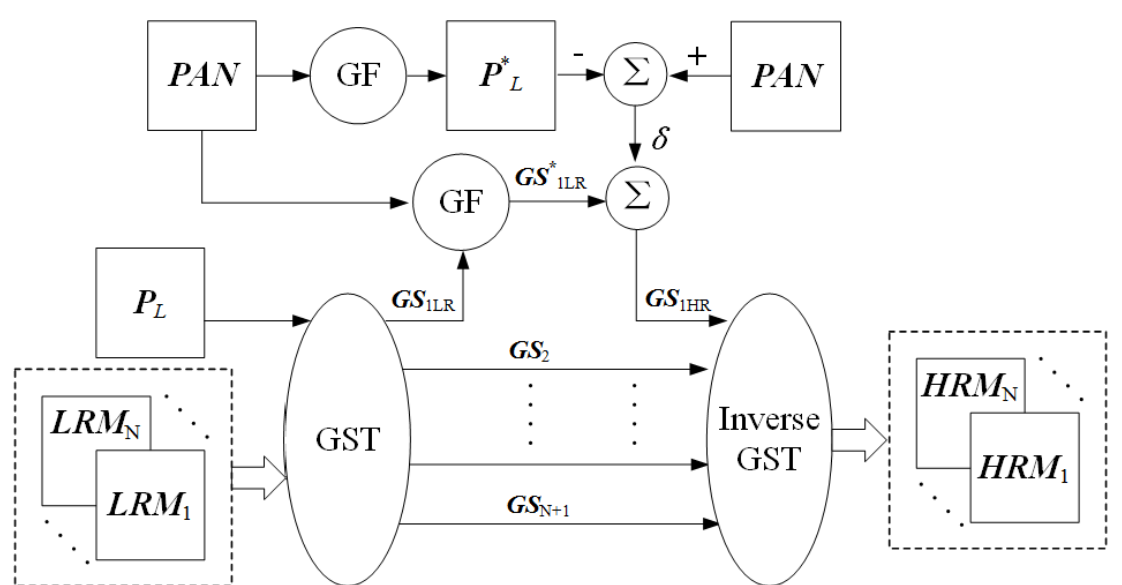

Figure 1. Schematic diagram

2) Simulate the lower spatial resolution PAN $\left(\boldsymbol{P}_{\boldsymbol{L}}\right)$ image by averaging all the resampled MS bands and set the $\boldsymbol{P}_{\mathrm{L}}$ together with all the $\boldsymbol{L} \boldsymbol{R} \boldsymbol{M}$ s as the input for GST, which is the same as the GS method.

3) Perform forward GST on the input images and obtain the transform bands $\left(\boldsymbol{G} \boldsymbol{S}_{1 \mathrm{LR}}, \boldsymbol{G} \boldsymbol{S}_{2} \ldots \boldsymbol{G} \boldsymbol{S}_{\mathrm{N}}, \boldsymbol{G} \boldsymbol{S}_{\mathrm{N}+1}\right)$.

4) Use the guided filter to extract the details from the PAN. We make the PAN image as the guidance image and the filtering input at the same time to produce the low-resolution version of the PAN image $\left(\boldsymbol{P}_{L}^{*}\right)$.

$$
\boldsymbol{P}_{L}^{*}=G F_{r, \varepsilon}(\boldsymbol{P} \boldsymbol{A N}, \boldsymbol{P} \boldsymbol{A N})
$$

Then the details of PAN image (denoted as $\boldsymbol{\delta}$ ) can be obtained by subtracting the $\boldsymbol{P}_{L}^{*}$ from the $\boldsymbol{P} \boldsymbol{A} \boldsymbol{N}$.

$$
\boldsymbol{\delta}=\boldsymbol{P} \boldsymbol{A N}-\boldsymbol{P}_{L}^{*}
$$

5) Use the guided filtering to transfer the structures of the $\boldsymbol{P} \boldsymbol{A N}$ to the $\boldsymbol{G} \boldsymbol{S}_{1 \mathrm{LR}}$ by

$$
\boldsymbol{G S _ { 1 L R } ^ { * }}=G F_{r, \varepsilon}\left(\boldsymbol{P A N}, \boldsymbol{G S} \boldsymbol{S}_{1 L R}\right)
$$

Experimentally we obtained the satisfied results when the radius $r=4$ and $\varepsilon=0.8$.

6) Generate a synthetic GS band $\left(\boldsymbol{G} \boldsymbol{S}_{1 \mathrm{HR}}\right)$ with high spatial resolution by injecting the details $\boldsymbol{\delta}$ into the $G S_{1 L R}^{*}$

$$
\boldsymbol{G S}_{1 H R}=\boldsymbol{\delta}+\boldsymbol{G} \boldsymbol{S}_{1 L R}^{*}
$$

7) Perform the inverse GST on the new transform bands $\left(\boldsymbol{G} \boldsymbol{S}_{1 \mathrm{HR}}, \boldsymbol{G S _ { 2 }}, \ldots, \boldsymbol{G} \boldsymbol{S}_{\mathrm{N}}, \boldsymbol{G S _ { \mathrm { N } + 1 }}\right)$ to produce the high spatial resolution MS images $\left(\boldsymbol{H} \boldsymbol{R} \boldsymbol{M}_{1}, \ldots, \boldsymbol{H} \boldsymbol{R} \boldsymbol{M}_{\mathrm{N}}\right)$.

\section{Experimental results}

\subsection{Test data and evaluation index}

The proposed method is evaluated on one coregistered data set acquired by GeoEye-1 satellite. The data set consists of 4-band MS imagery with $2.0 \mathrm{~m}$ spatial resolution and one PAN image with $0.5 \mathrm{~m}$ spatial resolution. The data set taken in Feb. 2009 covers the part area of Hobart, Australia. The original size of MS imagery is $3382 \times 7906$ pixels. Fig. 2 shows a subscene of $400 \times 400$ pixels with vegetation, cars, buildings, roads, and soil regions. Fig. 2(a) shows the PAN image and Fig. 2(b) reports the MS image.

In this paper, several commonly known objective quality indexes, including correlation coefficient (CC) [4], spatial CC (SCC) [5], spectral angle mapper (SAM) [6], Q4 [7], and QNR [8] are adopted to evaluate the sharpened products. QNR uses the original MS and PAN images as the references, and the other indexes use the bicubic interpolated MS image as the reference. We also implement and test GS, GSA, PRACS [9], PDI [10], APS [11], and MGF [12] methods for the purpose of comparison.

\subsection{Subjective analysis}

Figs. 2(c)-(i) show the sharpened results of the proposed, GS, GSA, PRACS, PDI, APS, and MGF method respectively. For the vegetation area, e.g. the green grass part, APS and MFG methods produce the significant color distortions, which may suffer from the inappropriate details injection. For the high reflection areas, such as the white roof of buildings, the color changes more or less from bright white to gray in GS, GSA, PRACS and MGF methods. PDI method (Fig. 2(g)) keeps spectral information well but the sharpness of the edges seems not enough compared with the PAN image (Fig. 2(a)). In terms of the sharpness, GS, GSA, APS and the proposed method produce the impressive results. It is clear to see that the proposed method obtains the high quality result. 


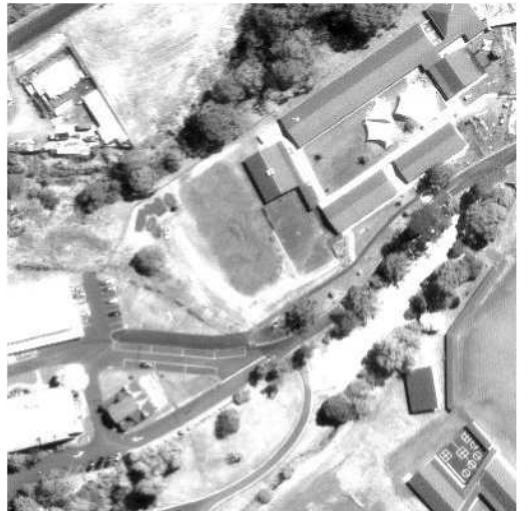

(a) PAN image

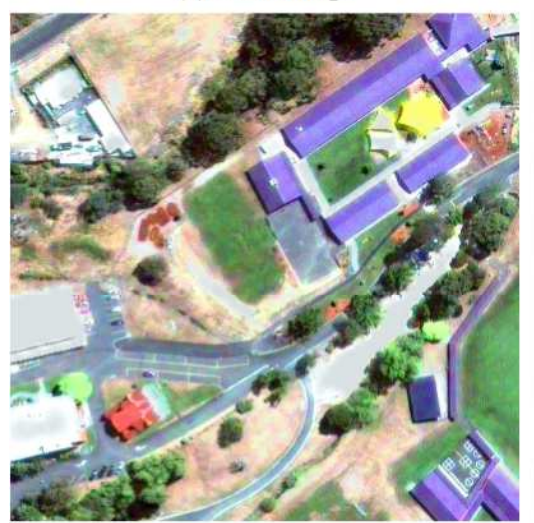

(d) GS method

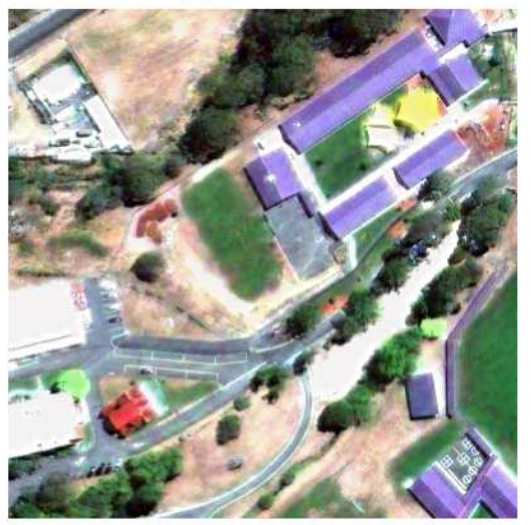

(g) PDI method

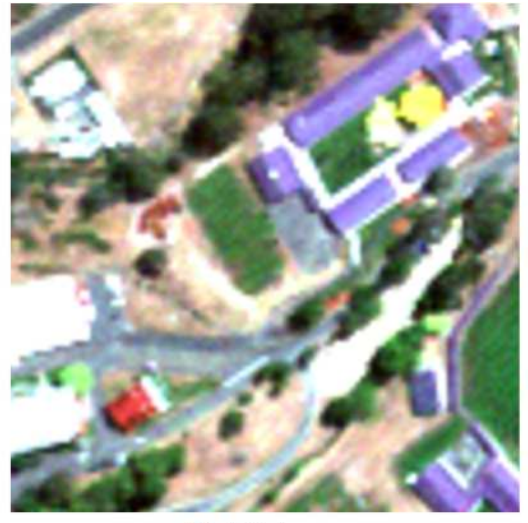

(b) MS image

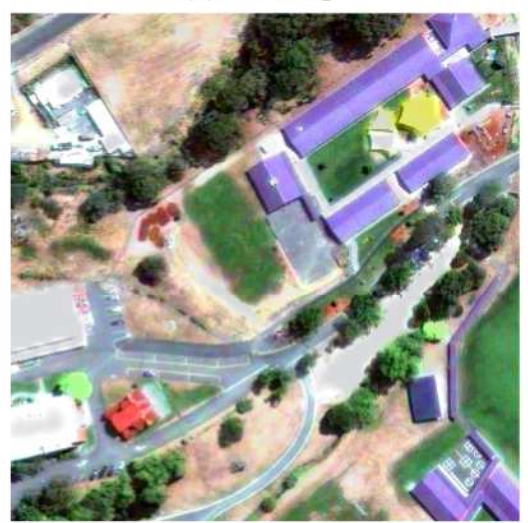

(e) GSA method

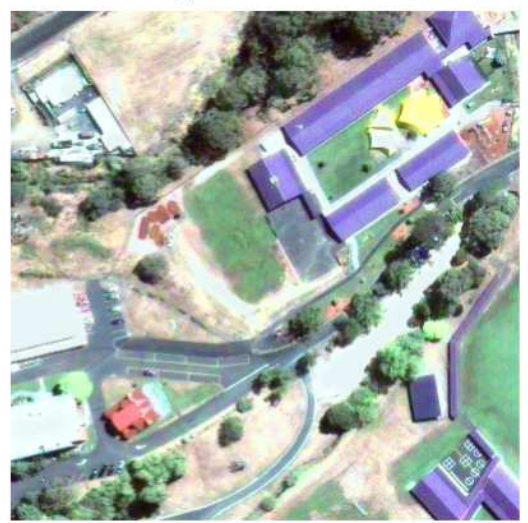

(h) APS method

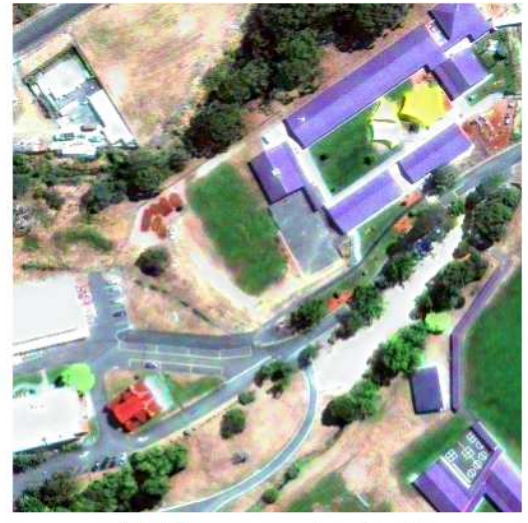

(c) The proposed method

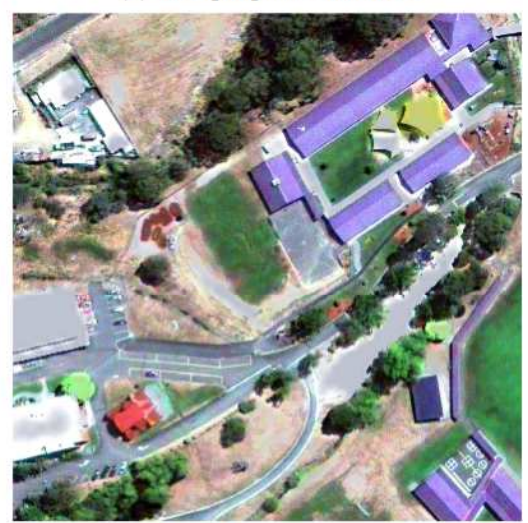

(f) PRACS method

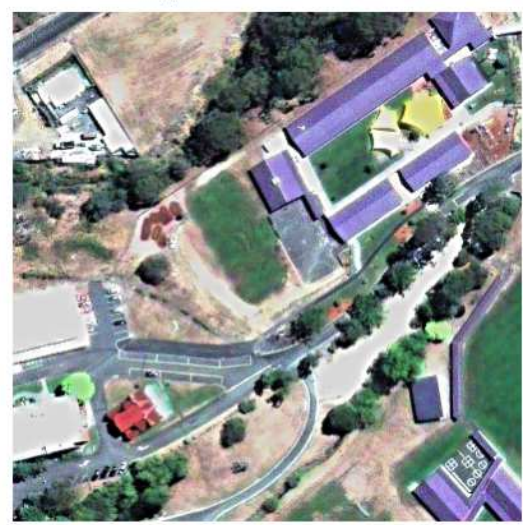

(i) MGF method

Figure 2. Test data and the pansharpened results

Fig. 2(c) appears the best among all the results, in which the color information is preserved well and the spatial details are as clear as the original PAN image.

\subsection{Objective analysis}

Table 1 reports the evaluation values for all the tested methods. Since CC and SCC indexes can be only applied to monochromatic image pair, the average value $\mathrm{CC}_{\mathrm{AVG}}\left(\mathrm{SCC}_{\mathrm{AVG}}\right)$ of all the sharpened $4 \mathrm{MS}$ bands is employed as a global spectral quality index. For the $\mathrm{SCC}_{\mathrm{AVG}}$ index, GS, GSA, APS, and the proposed method obtain excellent scores (all above 0.99), which means these methods can enhance the spatial resolution significantly. Our method stands second after APS method and the difference is very close (about 0.0013). However, APS produces more color distortions in terms of the other indexes. For the evaluations on the spectral distortion, MGF and APS method fall down to the last two, which means these two methods suffer severe color distortions and the result is consistent with the visual analysis. GS and GSA method are comparative in terms of $\mathrm{CC}_{\mathrm{AVG}}$ and QNR, and they are better than PRACS and PDI method according to Q4 and QNR indexes. The best scores of $\mathrm{CC}_{\mathrm{AVG}}$, SAM, Q4, and QNR all belong to the proposed method, which demonstrate that our method provides better performance and produces high-quality sharpened result. 
Table 1. Quality scores of the pansharpened results

\begin{tabular}{|l|c|c|c|c|c|c|c|}
\hline & GS & GSA & PRACS & PDI & APS & MGF & Proposed \\
\hline CC $_{\text {AVG }}$ & 0.9553 & 0.9558 & 0.9304 & 0.9564 & 0.9249 & 0.8873 & 0.9635 \\
\hline SCC $_{\text {AVG }}$ & 0.9942 & 0.9908 & 0.9661 & 0.9422 & 0.9959 & 0.9099 & 0.9946 \\
\hline SAM & 5.1068 & 4.8017 & 4.5084 & 3.9801 & 9.7581 & 6.0176 & 3.5186 \\
\hline Q4 & 0.8537 & 0.8063 & 0.7914 & 0.7959 & 0.7908 & 0.6751 & 0.8611 \\
\hline QNR & 0.8077 & 0.8041 & 0.7536 & 0.7278 & 0.7049 & 0.7015 & 0.8246 \\
\hline
\end{tabular}

\section{Conclusion}

In this paper we propose a new and effective Gram-Schmidt based pansharpening method. Using the guided filter, the method extracts the sufficient details from the panchromatic image and transfers the proper structures to the GS transform band. Moreover, the proposed method presents more freedom to sharpen the satellite images without considering the relative spectral responses among the imaging sensors. Experiments show that our method improves the existing GA and GSA methods and produces a satisfied high-quality pansharpened result.

\section{Acknowledgement}

The work is supported by the National Natural Science Foundation of China (No. 61301195), the Seed Foundation of Innovation and Creation for Graduate Students in Northwestern Polytechnical University, and Marie Curie International Research Staff Exchange Scheme Fellowship with the $7^{\text {th }}$ European Community Framework Programme (FP7) via LIVCODE (295151) and HAZCEPT (3187907).

\section{References}

[1] C. A. Laben and B. V. Brower. Process for enhancing the spatial resolution of multispectral imagery using Pansharpening. U.S. Patent 6011875, Jan. 4, 2000.

[2] B. Aiazzi, S. Baronti, and M. Selva. Improving component substitution pansharpening through multivariate regression of MS+Pan data. IEEE Transactions on Geoscience and Remote Sensing, 2007: 3230 - 3239.

[3] K. He, J. Sun, and X. Tang. Guided image filtering. IEEE Transactions on Pattern Analysis and Machine Intelligence, 2013: 1397 -1409.

[4] J. Marcello, A. Medina, and F. Eugenio. IEEE Geoscience and Remote Sensing Letters, 2013: 432 - 436.

[5] K. A. Kalpoma, K. Kawano, and J. Kudoh. IKONOS image fusion process using steepest descent method with bilinear interpolation. International Journal of Remote Sensing, 2013: 505 - 518.

[6] R. H. Yuhas, A. F. Goetz, and J. W. Boardman. Discrimination among semi-arid landscape endmembers using the spectral angle mapper (SAM) algorithm. Summaries 3 ${ }^{\text {rd }}$ Annual JPL Airborne Geoscience Workshop. 1992: 147 - 149.

[7] L. Alparone, S. Baronti, A. Garzelli, et al. A global quality measurement of pan-sharpened multispectral imagery. IEEE Geoscience and Remote Sensing Letters, 2004: 313 - 3167.

[8] L. Alparone, B. Aiazzi, S. Bareonti, et al. Multispectral and panchromatic data fusion assessment without reference. Photogrammetric Engineering \& Remote Sensing, 2008: 193 - 200.

[9] J. Choi, K. Yu, and Y. Kim. A new adaptive component-substitution-based satellite image fusion by using partial replacement. IEEE Transactions on Geoscience and Remote Sensing, 2011: 295 - 309.

[10] D. M. Zhang and X. D. Zhang. Pansharpening through proportional detail injection on generalized relative spectral response. IEEE Geoscience and Remote Sensing Letters, 2011: 978 - 982.

[11] T. Tu, C. L. Hsu, P. Y. Tu, et al. An adjustable pan-sharpening approach for IKONOS/QuickBird/GeoEye1/WorldView-2 imagery. IEEE Selected Topics in Applied Earth Observations and Remote Sensing, 2012: 125 - 134.

[12] K. P. Upla, S. Joshi, M. V. Joshi, et al. Multiresolution image fusion using edge-preserving filters. Journal of Applied Remote Sensing, 2015: 1 - 26. 